\title{
12 Lead Placement Mason Likar Modified
}

National Cancer Institute

\section{Source}

National Cancer Institute. 12 Lead Placement Mason Likar Modified. NCI Thesaurus.

Code C71110.

An electrocardiogram (ECG) lead placement whereby 12 leadpoints are recorded but the Mason Likar lead positions have been modified so that V1 to V 6 on the chest are part of a single electrode pad. In addition, lead CM5 is substituted for lead aVR. 